\title{
DENERVATION-INDUCED DECREASE IN MITOCHONDRIAL CALCIUM TRANSPORT IN RAT HIPPOCAMPUS ${ }^{1}$
}

\author{
MICHEL BAUDRY," CHRISTINE GALL, MARKUS KESSLER, HOSSIEN ALAPOUR, AND GARY LYNCH
}

Departments of Psychobiology and Anatomy, University of California, Irvine, California 92717

Received May 20, 1982; Revised August 18, 1982; Accepted August 24, 1982

\begin{abstract}
Calcium accumulation by mitochondria and the activity and in vitro phosphorylation of pyruvate dehydrogenase were measured in control and partially denervated hippocampus. Calcium uptake was measured with a calcium-sensitive electrode and ${ }^{45} \mathrm{Ca}^{2+}$ accumulation; both methods indicated that lesions of the entorhinal cortex produced a sizable reduction of calcium transport when mitochondria were fueled with pyruvate while much smaller changes were observed using succinate or ATP as energy sources. The decrease in calcium transport was evident by $24 \mathrm{hr}$ after the lesion and was still present 6 months later. Synaptic and nonsynaptic mitochondria were similarly affected by the lesions. The activity and in vitro phosphorylation of pyruvate dehydrogenase were also significantly reduced following lesions of the entorhinal cortex, suggesting that denervation altered the endogenous state of phosphorylation of the mitochondrial enzyme. Commissural lesions but not septal lesions also resulted in a decrease in mitochondrial calcium transport when mitochondria were fueled with pyruvate.

These findings suggest that denervation disturbs mitochondrial regulation of free calcium via an action on enzymes which regulate pyruvate dehydrogenase phosphorylation and activity. The potential relationship of this effect to degenerative changes associated with deafferentation and certain disease states is discussed.
\end{abstract}

Neuronal degeneration and atrophy in brain are found in a number of disease states and quite possibly form part of the aging process. Although little is known of the biochemistry involved in these pathologic states, an increasing body of evidence points to a role for calcium and calcium-activated proteinases in experimentally induced degeneration in the peripheral nervous system (Schlaepfer, 1977; Schlaepfer and Micko, 1978). It becomes of interest therefore to determine if atrophic effects in brain are preceded by perturbations in calcium-buffering mechanisms. Toward this end, we recently investigated the effects of denervation on calcium sequestration by mitochondria in hippocampus. Mitochondria possess a high-affinity, large capacity uptake system for calcium and it has become increasingly apparent that they play an important role in buffering calcium levels inside neurons (Bygrave, 1977; Erulkar and Fine, 1979). The hip-

\footnotetext{
'This work was supported by Grants NIA AG00538 and NIMH MH 19793-11 to G. L. and NIH NS 18427-01 to M. B.

We wish to thank Dr. J. Fuchs and D. Arst for their participation in the initial experiments of the study and Karen Zfaty for the preparation of this manuscript.

${ }^{2}$ To whom correspondence should be addressed at Department of Psychobiology, University of California, Irvine, California 92717.
}

pocampus was selected for analysis because the dendritic shrinkage, spine losses, and reactive growth responses which occur in this structure following destruction of its primary afferents have been both quantitatively and qualitatively described (Rose et al., 1976; Gall et al., 1978, 1979, 1980).

In our first study on denervation and calcium regulation, we compared pyruvate versus succinate- or ATPsupported calcium sequestration by hippocampal mitochondria after lesions of the entorhinal cortex. Pyruvate is the primary source of carbon for the tricarboxylic acid cycle (via its conversion to acetyl-CoA) and the activity of pyruvate dehydrogenase ( $\mathrm{PDH})$ is linearly related to calcium uptake by brain mitochondria (Browning et al., 1981), making it more likely that changes in pyruvatedependent functions will have more important consequences than changes in succinate- or ATP-dependent calcium transport. We found that PDH activity and pyruvate-fueled uptake were substantially depressed in mitochondria isolated from denervated hippocampi; ATP-supported uptake was not detectably changed under these conditions (Baudry et al., 1982a).

The experiments described in the present report exlend these initial observations by first determining the time course of the depression of uptake and, second, 
establishing if removal of other major afferents of hippocampus produces effects similar to those observed after entorhinal lesions. In addition, we sought to test the idea that the level to which mitochondria are able to buffer free calcium concentrations (the "set-point") is affected by prior denervation. The results are discussed in terms of a hypothesis of degenerative diseases of the CNS.

\section{Materials and Methods}

Male Sprague-Dawley rats (150 to $250 \mathrm{gm}$ ) were maintained in a well-ventilated environment at a constant temperature of $22^{\circ} \mathrm{C}$ with a light-dark cycle (lights on 7 A.M. to 7 P.M.) and with free access to food and water.

\section{Lesions of various afferent pathways}

Removal of the commissural afferents to the hippocampal formation was accomplished by the aspiration of the contralateral hippocampal formation (with special care taken to not damage the septum ipsilateral to the lesion). The septal and entorhinal afferents to the hippocampal formation were eliminated by making stereotaxically placed electrolytic lesions in the ipsilateral medial septal nucleus and nucleus of the diagonal bands and in the ipsilateral entorhinal cortex, respectively. All lesions were made with the rats under sodium pentobarbital anesthesia. Electrolytic lesion coordinates were verified histologically prior to their use on the experimental animals (see Fig. 1).

\section{Preparation of mitochondria}

Crude mitochondrial fraction. Rats were killed by decapitation, and the hippocampi was rapidly dissected and homogenized in $2 \mathrm{ml}$ of cold $0.32 \mathrm{~m}$ sucrose. Crude mitochondrial fractions were prepared by differential centrifugation (first centrifugation $1,000 \times g$ for $10 \mathrm{~min}$ followed by centrifugation of the supernatant at 14,000 $\times g$ for $20 \mathrm{~min}$ ).

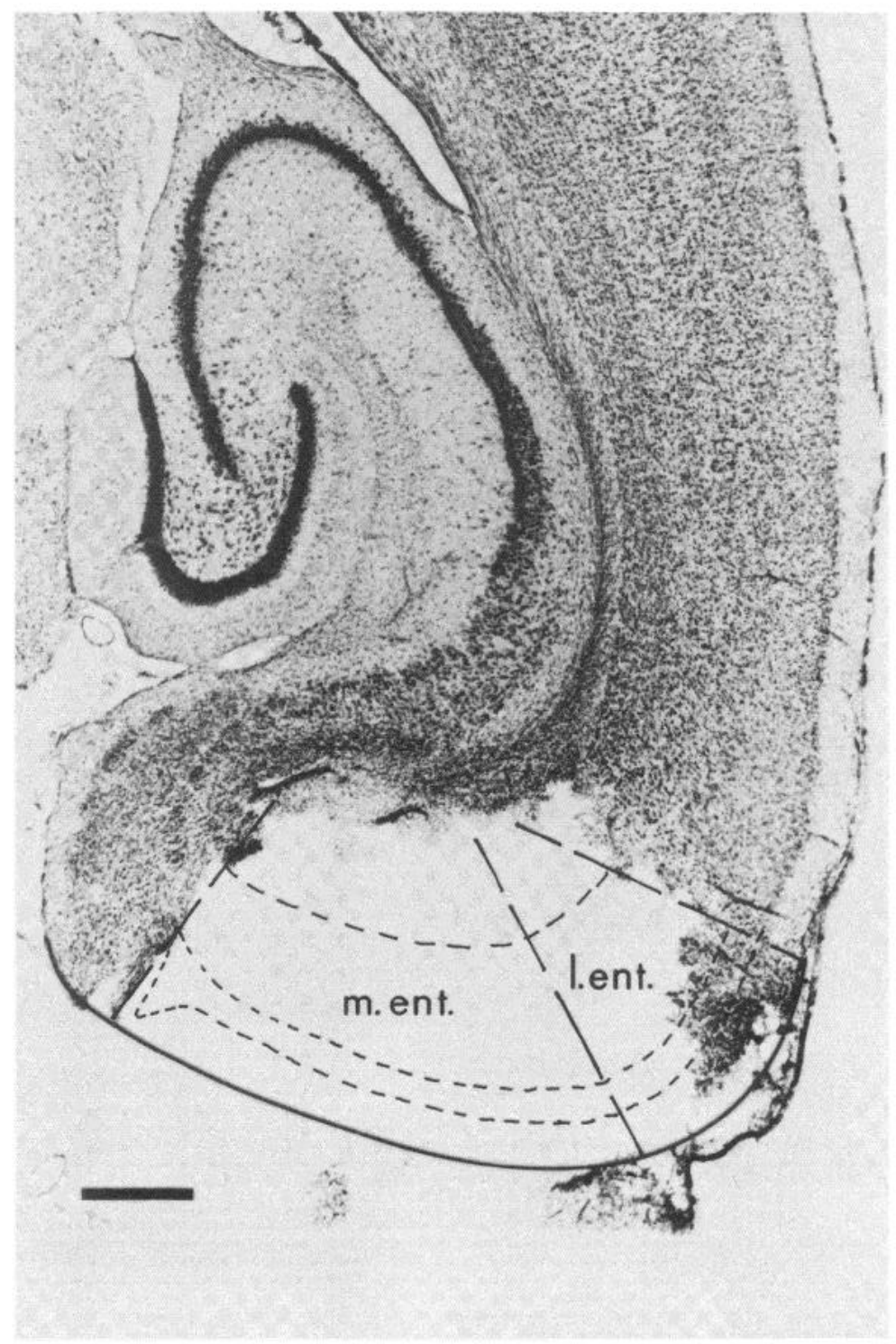

Figure 1. Histologic verification of the entorhinal cortex lesions. This Nissl-stained horizontal section through the hippocampal formation and retrohippocampal area of an adult rat illustrates the extent of an electrolytic lesion of the entorhinal cortex placed by the same coordinates used in the biochemical studies. $m$. ent., medial entorhinal cortex; $l$. ent., lateral entorhinal cortex. Calibration bar: $300 \mu \mathrm{m}$. 
Purified mitochondria. Purified mitochondria were prepared according to the method of Clark and Nicklas (1970) as previously described (Browning et al., 1981). The separation of free and synaptic mitochondria was obtained according to the method of Lai and Clark (1976).

\section{Calcium accumulation}

The various mitochondrial fractions were reconstituted in a buffer consisting of $150 \mathrm{~mm} \mathrm{KCl}, 1.3 \mathrm{~mm}$ $\mathrm{MgSO}_{4}, 2.4 \mathrm{~mm} \mathrm{NaH} \mathrm{PO}_{4}, 20 \mathrm{~mm}$ HEPES, and sufficient Tris to adjust the $\mathrm{pH}$ to 6.6 .

${ }^{45} \mathrm{Ca}$ accumulation. The accumulation of ${ }^{45} \mathrm{Ca}(100 \mathrm{Ci} /$ mol; International Chemical and Nuclear Pharmaceuticals, Irvine, CA) was measured as previously described (Browning et al., 1981) at a fixed calcium concentration of about $2 \mu \mathrm{M}$, obtained with the use of a calcium-EGTA buffer.

Calcium accumulation measured with a calcium-sensitive electrode. Aliquots of the mitochondrial fractions were preincubated for $5 \mathrm{~min}$ at room temperature $(0.1$ to $0.2 \mathrm{mg}$ protein $/ \mathrm{ml}$ ) in a final volume of $5 \mathrm{ml}$ in the presence of $1 \mathrm{mM}$ ADP (since the omission of $0.1 \mathrm{mM}$ CoA did not modify the properties of calcium transport, it was omitted in all of the expcriments). Free calcium was monitored by a calcium-sensitive electrode (Beckman, Irvine, CA) and millivolts were recorded on an Omniscribe recorder. The electrode was calibrated using calcium EGTA buffers by applying the formula $[\mathrm{Ca}]^{2+}$ free $=K R /(1-R)$ where $R$ represents the ratio of total calcium over total EGTA and $\mathrm{K}$ the dissociation constant for calcium-EGTA which equals $1.28 \times 10^{-6} \mathrm{M}$ at $\mathrm{pH} 6.6$ (Portzehl et al., 1964).

\section{PDH activity and phosphorylation}

Crude mitochondrial fractions were resuspended by sonication in cold $25 \mathrm{~mm}$ PIPES buffer $(\mathrm{pH} 7.2)$ containing 1 mM EDTA. "Active" and "total" PDH activity were measured as described previously (Baudry et al., 1982a), whereas $\alpha$-PDH phosphorylation was determined by a modification of the method of Browning et al. (1981) as described elsewhere (Baudry et al., 1982b).

Proteins were determined according to the method of Lowry et al. (1951) with bovine serum albumin as standard.

Student's $t$-test was used to statistically compare various data; in the case of ipsilateral versus contralateral side comparisons, the paired $t$-test was used.

\section{Results}

\section{${ }^{45} \mathrm{Ca}$ accumulation by hippocampal mitochondria at various times following a unilateral entorhinal cortex lesion}

We measured ${ }^{45} \mathrm{Ca}$ accumulation in the mitochondrial fraction from the ipsilateral (denervated) and contralateral (control) hippocampus at various times following a unilateral lesion of the entorhinal cortex using pyruvate, succinate, or ATP as substrates (Fig. 2). The pyruvatesupported accumulation in the ipsilateral mitochondria was significantly decreased $24 \mathrm{hr}$ after the lesions; the effect reached a maximum 5 days after the lesions, at which point it represented a $45 \%$ decrease. A slight

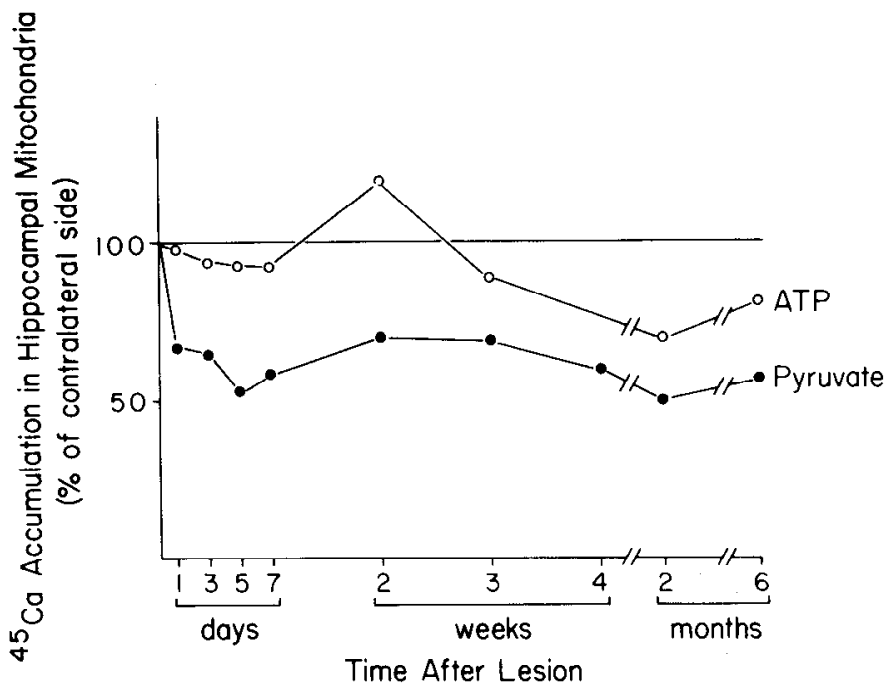

Figure 2. Changes in pyruvate- and ATP-supported calcium transport at various times after unilateral entorhinal cortex lesions. ${ }^{45} \mathrm{Ca}$ Accumulation supported by pyruvate or ATP in hippocampal crude mitochondrial fractions was measured at various times following unilateral entorhinal cortex lesions as described under "Materials and Methods." Results, which are the means of three to six experiments, are expressed as percentage of the values found in the contralateral side.

TABLE I

Changes in the ratio of $\mathrm{P} / \mathrm{S}$ - and $\mathrm{P} / \mathrm{A}$-supported ${ }^{45} \mathrm{Ca}$ accumulation at various times after a unilateral entorhinal cortex lesion

At various times following a unilateral entorhinal cortex lesion, rats were killed by decapitation, the hippocampi were rapidly dissected, and crude mitochondrial fractions were prepared as described under "Materials and Methods." Pyruvate-, succinate-, and ATP-supported ${ }^{45} \mathrm{Ca}$ accumulations were measured in the lesioned ipsilateral side and the control contralateral side. Ratios of P/S- or P/A-supported calcium transport were calculated. The results represent the percentage of decrease in the ipsilateral side as compared with the contralateral side and are mean $\pm \mathrm{SE}$. of the indicated number of experiments (in parentheses). Paired $t$-test was used to evaluate statistically the levels of significance of the differences, and in every case $p$ was found at least $<0.05$.

\begin{tabular}{lccccc}
\hline & \multicolumn{5}{c}{ Time After the Lesion } \\
\cline { 2 - 6 } & 1 day (3) & 3 days (3) & 1 week (12) & 3 weeks (3) & 6 months (6) \\
\hline $\mathrm{P} / \mathrm{S}$ & $-20 \pm 8$ & $-34 \pm 10$ & $-30 \pm 5$ & $-19 \pm 6$ & $-28 \pm 7$ \\
$\mathrm{P} / \mathrm{A}$ & $-32 \pm 9$ & $-26 \pm 17$ & $-38 \pm 6$ & $-24 \pm 12$ & $-27 \pm 13$ \\
\hline
\end{tabular}

tendency to return to control values was observed between 5 days and 2 weeks after the lesions, after which uptake declined to minimal values and remained at this level. No significant decrease in the ATP-supported ${ }^{45} \mathrm{Ca}$ accumulation was detected before 3 weeks, after which uptake decreased by about $15 \%$. These data can be further analyzed by calculating the ratios of the pyruvateover succinate- $(\mathrm{P} / \mathrm{S})$ or pyruvate- over ATP $(\mathrm{P} / \mathrm{A})$ supported ${ }^{45} \mathrm{Ca}$ accumulations, values which are independent of the amount of protein and therefore eliminate the possibility that the observed changes reflect differences in the amount of mitochondria in the various samples (Table I). These ratios were significantly decreased by 25 to $30 \%$ as soon as 1 day after the lesion and remained decreased at each time point after the lesion. 


\section{Effects of unilateral entorhinal cortex lesion on ${ }^{45} \mathrm{Ca}$ accumulation in free and synaptic hippocampal mitochondria}

To determine whether the changes in ${ }^{45} \mathrm{Ca}$ accumulation by mitochondrial fractions occur more specifically in free or synaptic mitochondria, we separated these two categories of mitochondria according to the method of Lai and Clark (1976). One week after a unilateral entorhinal cortex lesion, the pyruvate-supported ${ }^{45} \mathrm{Ca}$ accumulation was similarly reduced by about $55 \%$ in both free and synaptic mitochondria (Table II). The succinatesupported ${ }^{45} \mathrm{Ca}$ accumulation was also slightly but significantly reduced, whereas the ATP-supported ${ }^{45} \mathrm{Ca}$ accumulation was not significantly modified. Thus, the ratios pyruvate- over succinate- or pyruvate- over ATPsupported calcium transport were also similarly reduced in free and synaptic mitochondria.

\section{Effects of lesions to various afferent pathways to the hippocampus on calcium transport by hippocampal mitochondria}

The major extrinsic afferents of inputs to hippocampus arise in the ipsilateral entorhinal cortex and the contralateral hippocampus. The septum, thalamus, and brainstem provide projections which generate far smaller populations of synapses. We measured the effects of commissural lesions on the pyruvate-, succinate- , or ATPsupported calcium transport by hippocampal mitochondria 1 week after a unilateral hippocampal aspiration (Table III). The pyruvate-supported calcium was significantly reduced by $25 \%$ in the mitochondrial fraction prepared from contralateral hippocampus as compared with unoperated rats. The succinate- or the ATP-supported calcium transport was not modified by the lesion. Consequently, the ratios of $\mathrm{P} / \mathrm{S}$ or $\mathrm{P} / \mathrm{A}$ were also significantly reduced by $25 \%$. On the other hand, unilateral lesions of the septum produced no significant effects on pyruvate-, succinate- , or ATP-supported calcium transport of mitochondria from the ipsilateral hippocampus as compared with the contralateral hippocampus (data not shown).

\section{TABLE II}

Changes in pyruvate-, succinate-, and ATP-supported calcium transport in free and synaptic mitochondria 1 week after a unilateral entorhinal cortex lesion

One week after a unilateral entorhinal cortex lesion, rats were killed by decapitation, their hippocampi were rapidly dissected, and free and synaptic mitochondria were prepared as described under "Materials and Methods." Results representing the values found in the ipsilateral side preparation are expressed as percentage of the values found in the contralateral unlesioned side and are mean $\pm \mathrm{SE}$ of six experiments.

\begin{tabular}{lcl}
\hline & $\begin{array}{c}\text { Synaptic } \\
\text { Mituchundria }\end{array}$ & $\begin{array}{c}\text { Free } \\
\text { Mitochondria }\end{array}$ \\
\hline $\mathrm{P}$ & $-53 \pm 10 \%^{a}$ & $-56 \pm 6 \%^{a}$ \\
$\mathrm{~S}$ & $-25 \pm 13 \%^{b}$ & $-23 \pm 10 \%^{b}$ \\
$\mathrm{~A}$ & $-3 \pm 14 \%^{\circ}$ & $-14 \pm 11 \%^{c}$ \\
$\mathrm{P} / \mathrm{S}$ & $-38 \pm 11 \%^{a}$ & $-42 \pm 6 \%^{a}$ \\
$\mathrm{P} / \mathrm{A}$ & $-53 \pm 7 \%^{a}$ & $-50 \pm 3 \%^{a}$ \\
\hline
\end{tabular}

${ }^{\alpha} p<0.001$ (paired $t$-test).

$p<0.01$.

Not significant.
TABLE III

Effect of commissural lesions on pyruvate-, succinate-, and ATPsupported calcium transport on hippocampal mitochondria

One week after a unilateral hippocampal aspiration, rats were killed by decapitation, the hippocampi were rapidly dissected, and crude mitochondrial fractions were prepared as described under "Materials and Methods." The pyruvate-, succinate-, and ATP-supported ${ }^{45} \mathrm{Ca}$ accumulation were measured in the contralateral side (lesion) and compared with values obtained in control nonoperated animals. Results for the pyruvate-, succinate-, or ATP-supported calcium transport are expressed in nanograms of calcium/milligrams of protein and are mean $\pm \mathrm{SE}$. Number of experiments in parentheses.

\begin{tabular}{lccc}
\hline & Control (14) & Lesion (14) & $\%$ Change \\
\hline P & $519 \pm 50$ & $387 \pm 27$ & $-25^{a}$ \\
S & $1440 \pm 85$ & $1399 \pm 88$ & $-3^{b}$ \\
ATP & $694 \pm 40$ & $685 \pm 50$ & $-1^{b}$ \\
P/S & $0.36 \pm 0.02$ & $0.28 \pm 0.02$ & $-22^{a}$ \\
P/A & $0.75 \pm 0.07$ & $0.56 \pm 0.05$ & $-25^{a}$
\end{tabular}

" $p<0.05$ (Student's $t$-test).

"Not significant.

Effects of entorhinal cortex lesions on calcium transport by hippocampal mitochondria studied with a calcium-sensitive electrode

Calcium transport in control animals. In order to define further how the decrease in pyruvate-supported ${ }^{45} \mathrm{Ca}$ accumulation could modify the regulation of calcium buffering inside the cell, we investigated the effects of entorhinal cortex lesions on hippocampal calcium transport using a calcium-sensitive electrode. This technique has shown that mitochondria buffer free calcium to the low micromolar range (Becker et al., 1980; Nicholls and Scott, 1980). We first showed that brain mitochondria were also able to decrease free calcium levels in the low micromolar range (about $0.5 \mu \mathrm{M}$ ) using pyruvate as a substrate (Fig. 3). Once a plateau is reached and a pulse of calcium is given, the rate of transport decreases and the new set-point is reached at a higher calcium concentration. This is not due to an exhaustion of the substrate because adding more pyruvate does not modify the setpoint. This effect is not due to a saturation of the mitochondria because after adding succinate, mitochondria will reduce free calcium to lower concentrations than were observed with pyruvate alone (Fig. 3). The pyruvate-supported calcium transport in mitochondria is totally blocked by the inhibitor of pyruvate transport $\alpha$ cyanohydroxycinnamate $(0.1 \mathrm{~mm})$ and by the uncoupler dinitrophenol $(0.1 \mathrm{mM})$. Once equilibrium is reached, addition of the calcium ionophore $\mathrm{A}_{23} 187(5 \mu \mathrm{M})$ induces an immediate release of calcium into the medium. We first demonstrated that treatment resulting in a modification in the state of phosphorylation of $\alpha-\mathrm{PDH}$ could result in changes in calcium transport similar to those we previously reported using the ${ }^{45} \mathrm{Ca}$ accumulation method (Browning et al., 1981). As shown in Figure 3, preventing the phosphorylation of $\alpha$-PDH by preincubating mitochondria with an inhibitor of PDH kinase, dichloroacetate (DCA) (Leiter et al., 1978), resulted in an increased rate of calcium transport as well as in a decrease in the set-point at which mitochondria buffer free calcium $(0.35$ $\mu \mathrm{M}$ instead of $0.55 \mathrm{~mm}$ ). It also reduced the rate of inactivation of the pyruvate-supported calcium transport 


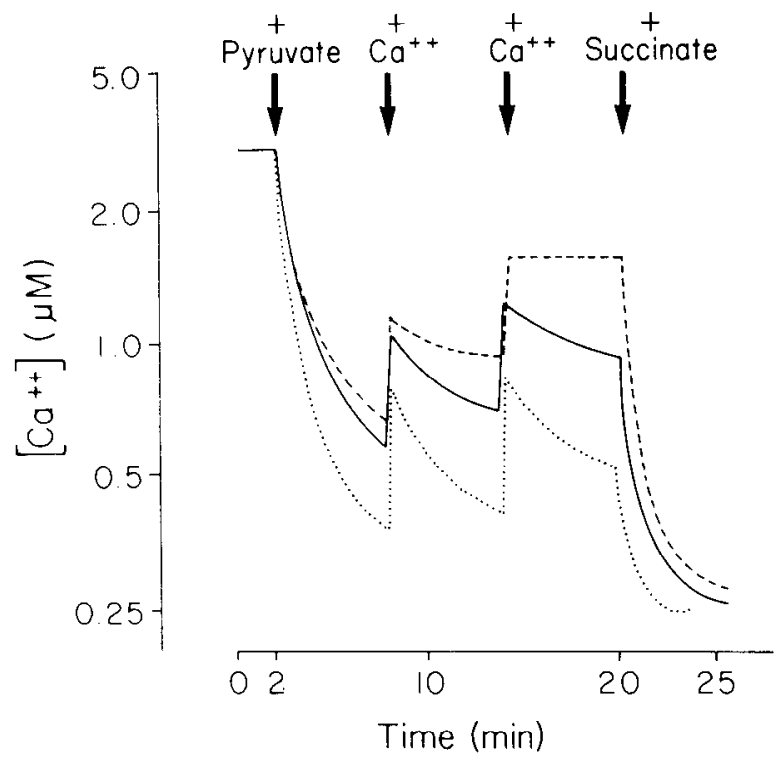

Figure 3. Effect of DCA and fluoride on the regulation of calcium transport in mitochondria. Forebrain mitochondria were purified as described under "Materials and Methods" and incubated at room temperature under control conditions $(\longrightarrow)$ or in the presence of $5 \mathrm{~mm}$ DCA $(\ldots \ldots)$ or $10 \mathrm{~mm}$ potassium fluoride (-- ). Free calcium concentration was measured with a calcium-sensitive electrode. After a 2-min preincubation, $0.5 \mathrm{~mm}$ pyruvate was added. At the indicated times, calcium chloride was added ( $25 \mathrm{nmol} / \mathrm{mg}$ of protein). Succinate ( $5 \mathrm{~mm}$ ) was added after $20 \mathrm{~min}$ of incubation. The experiment was replicated three times with similar results.

following successive additions of calcium. On the other hand, DCA had no effect on the succinate-supported transport whether the succinate is added after pyruvate addition (Fig. 3) or before (data not shown). Conversely, addition of fluoride ions, which have been shown to inhibit PDH phosphatase therefore preventing the dephosphorylation of PDH (Leiter et al., 1978), resulted in a decreased rate of transport as well as in increase in the set-point (Fig. 3). More significantly, it also resulted in an increase in the rate of inactivation of the pyruvatesupported calcium transport. This possibly reflects the fact that mitochondria have to generate enough ATP to phosphorylate $\alpha-\mathrm{PDH}$, which can occur only when calcium transport stops. In this case, too, addition of fluoride did not modify the succcinate-supported calcium transport.

Effects of unilateral entorhinal cortex lesions. One week after a unilateral entorhinal cortex lesion, the rate of calcium transport supported by pyruvate was reduced in the mitochondria prepared from the ipsilateral side as compared with the contralateral side. In addition, the set-point at which mitochondria buffer free calcium levels was significantly elevated (Fig. $4 A$ ). The rate of inactivation of the pyruvate-supported calcium transport was also markedly increased in the lesion side as compared with the control side. On the other hand, the succinatesupported calcium transport is only slightly decreased on the lesion side as compared with the control side (Fig. $4 B$ ). A similar effect was observed as early as 1 day after the lesion (data not shown).

\section{Effects of entorhinal cortex lesions on PDH activity and phosphorylation}

Since most of the previously reported data pointed to an effect of the lesion on the activity and/or the state of phosphorylation of $\mathrm{PDH}$, it was of interest to measure these two parameters. One week after the unilateral lesion of the entorhinal cortex, there was a small but significant decrease in the active $\mathrm{PDH}$ activity without changes in the total PDH activity, accompanied by a reduced in vitro phosphorylation of its $\alpha$-subunit (Table IV).

\section{Discussion}

The present data show that lesions of the major extrinsic inputs to the hippocampus result in marked modifications in the regulation of calcium transport by hip-

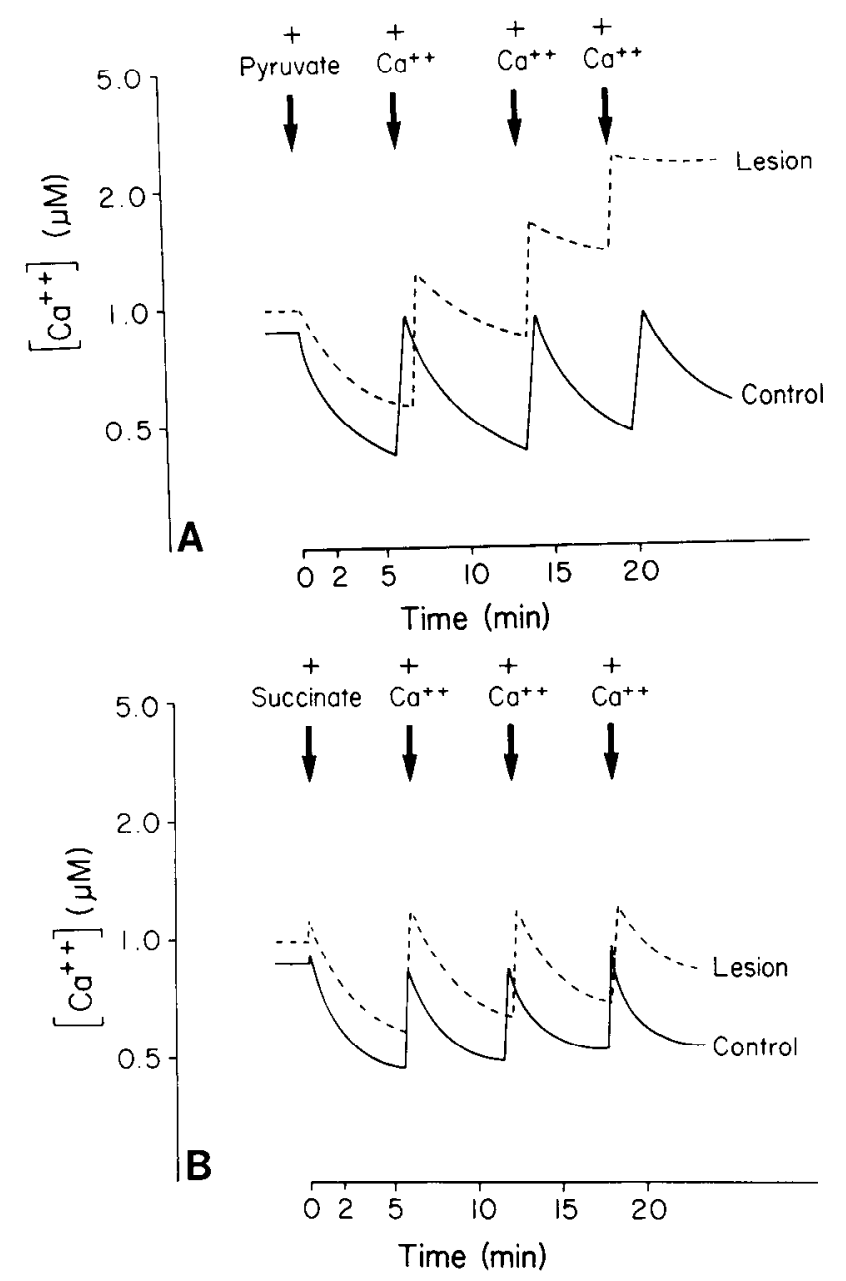

Figure 4. Changes in calcium transport by purified hippocampal mitochondria 1 week after unilateral entorhinal cortex lesion. Mitochondria from four pooled hippocampi of ipsilateral (lesion) or contralateral (control) sides were purified 1 week following unilateral entorhinal cortex lesions. Equal amounts of proteins $(0.2 \mathrm{mg}$ of protein $/ \mathrm{ml})$ from the control and lesioned preparations were preincubated at room temperature. Changes in calcium concentrations were measured with a calcium-sensitive electrode as described in "Materials and Methods." $A$, Pyruvate $(0.5 \mathrm{mM})$ and $\mathrm{Ca}^{2+}(25 \mathrm{nmol} / \mathrm{mg}$ protein) were added as indicated. $B$, Succinate $(5 \mathrm{~mm})$. The experiment was replicated three times with similar results. 
TABLE IV

Effects of unilateral entorhinal cortex lesions on $\mathrm{PDH}$ activity and phosphorylation

One week after a unilateral entorhinal cortex lesion, rats were killed by decapitation, their hippocampi were rapidly dissected, and crude mitochondrial fractions were prepared as described under "Materials and Methods." Active and total PDH activity data are from Baudry et al. (1982a). For $\alpha$-PDH phosphorylation, autoradiographs were analyzed by quantitative densitometry, and the data expressed as the ratio of the peak height of the PDH band to the total peak height of the other major bands (excluding the $50 \mathrm{~K}$ band which showed inconsistent variation among different experiments).

\begin{tabular}{ccc}
\hline Side & $\begin{array}{c}\text { PDH Activity } \\
\text { (\% active/total) }\end{array}$ & $\begin{array}{c}\alpha \text {-PDH } \\
\text { Phosphorylation }\end{array}$ \\
\hline Lesion (6) & $39 \pm 2^{a}$ & $56.9 \pm 1.3^{a}$ \\
& $-20 \%$ & $-16 \%$ \\
Control (6) & $49 \pm 2$ & $67.4 \pm 2.4$ \\
\hline
\end{tabular}

“ $p<0.01$ (Student's $t$-test).

pocampal mitochondria. With the use of two quite different techniques, we found that pyruvate-supported calcium transport was significantly reduced, while only small reductions occurred for succinate- or ATP-supported calcium transport. The effects of the lesion on pyruvate-supported calcium transport were evident as early as $24 \mathrm{hr}$ postlesion and persisted for at least 6 months after the lesion. Since shrinkage of the molecular layer and electron-opaque degeneration are not present $24 \mathrm{hr}$ after the lesion, changes in calcium regulation precede much if not all of the pathologic responses of the dendrites. This strengthens the possibility that perturbations in calcium regulation are causally linked to atrophic effects of denervation. The fact that calcium transport is decreased to the same extent in both synaptic and free mitochondria indicates that the effects of the lesions on calcium regulation are not restricted to a specific population of mitochondria and could occur in neuronal as well as glial elements. However, considering that glial elements represent a small fraction of the neuropil (McWilliams and Lynch, 1978), it is more likely that the bulk of the effect is localized in neuronal mitochondria.

Superimposed on the immediate effect of the lesion on pyruvate-supported transport, a delayed decrease in succinate- or ATP-supported uptake was also found after unilateral entorhinal cortex lesions.

The denervation-induced perturbations tended to be less pronounced during the period from 5 days to 3 weeks after the lesion. This is the time frame during which axon sprouting begins and reaches its maximum rate in the hippocampus (McWilliams and Lynch, 1979). This time course is also similar to that for metabolic alterations studied with the $\left[{ }^{3} \mathrm{H}\right] 2$-deoxyglucose autoradiography technique (Stewart and Smith, 1980). In that study, unilateral lesions of the entorhinal cortex resulted in a generalized decrease in 2-deoxyglucose uptake in the denervated territory beginning at 1 day postlesion and continuing for at least 1 month thereafter. However, between postlesion days 6 and 10, an increased 2-deoxyglucose uptake was observed in the outer molecular layer of the dentate gyrus.

Several arguments indicate that the effect of the lesion on mitochondrial calcium transport is mediated through an action on $\mathrm{PDH}$ phosphorylation and consequently $\mathrm{PDH}$ activity. The decrease in the ratio of active to total PDH activity, which has been shown to reflect the state of phosphorylation of the $\alpha$-subunit of the enzyme (Mitrius et al., 1981; Morgan and Routtenberg, 1981), was accompanied by a decrease of the same magnitude of the in vitro phosphorylation of the $\alpha$-subunit. Changes in active PDH were in turn highly correlated with changes in pyruvate-supported calcium transport (Baudry et al., 1982a). This is consistent with the idea that the $\alpha$-subunit is more phosphorylated on the lesion than the control side and that this is associated with a reduction in $\mathrm{PDH}$ activity. In agreement with this idea is the fact that inhibiting PDH dephosphorylation by fluoride mimicks the effects of the lesion on the pyruvate-supported calcium transport (compare Figs. 3 and $4 A$ ). This last result raises the possibility that the lesions decrease $\mathrm{PDH}$ phosphatase activity. In this regard it is noteworthy that insulin decreases PDH phosphorylation (and thereby increases PDH activity) by liberating a peptide intermediary which stimulates PDH phosphatase (Seals and Czech, 1980; Seals and Jarret, 1980). It is thus possible that the changes seen after lesions are reflections of the loss of a modulatory factor normally provided by the intact afferents.

The slower occurring, general decrease in calcium transport might be a result of a general alteration in metabolism previously reported in various target zones following denervation. For example, denervation or disuse induces a decrease in the levels of several mitochondrial enzymes and of calcium transport in muscles (Nemeth et al., 1981; Joffe et al., 1981). In the CNS, lesions of the noradrenergic or dopaminergic neurons similarly result in decreased oxidative metabolism in the target zones of these neurons (La Manna et al., 1981; Marshall et al., 1981). It is not clear whether these slower occurring changes are consequences of the initial disturbances in mitochondrial metabolism set up by the change in PDH activity or whether they represent a totally separate phenomenon.

The use of the calcium-sensitive electrode to study the kinetics of calcium transport by mitochondria served to define the potential consequences of denervation for target dendrites. Mitochondria, in the presence of appropriate substrates, sequester calcium until free concentration of the cation reaches very low values, defined as a set-point. At this concentration it is assumed that release of calcium is equilibrated with uptake. Presumably, ATP synthesis then takes place as long as calcium levels remain at this low value. Under these conditions, the mitochondria very rapidly accumulate any excess of free calcium as demonstrated by applying successive pulses of calcium. Increasing PDH activity by decreasing its phosphorylation state not only increases the rate at which calcium is transported but also decreases the value of the set-point, whereas increasing PDH phosphorylation has the opposite effects. Lesions of the entorhinal cortex result not only in an increase in the value of the set-point to which mitochondria buffer calcium but also in a marked increase in the rate of inactivation of the pyruvate-supported transport following successive addi- 
tions of calcium. This effect could considerably amplify the differences between the lesion and the control sides (see Fig. 4A). It is thus possible that calcium levels increase markedly in the cytoplasm of the deafferented cells as a result of successive influxes of calcium. Indeed, it has been reported that calcium levels are substantially increased in the spinal cord following trauma (Happel et al., 1981).

Elevations in internal calcium can be expected to activate a variety of normally quiescent calcium-dependent processes in the target cells. Neurons are known to contain calcium-sensitive proteinases (Ishiura, 1981) which are similar if not identical to those thought to initiate degenerative changes in peripheral axons (Schlaepfer, 1979). We propose therefore that activation of such processes in the denervated dendrites is responsible for the initial atrophy which follows denervation. In this context, it is of considerable interest that alterations in $\mathrm{PDH}$ activity have been reported to occur in several degenerative diseases of the CNS. Decreases in $\mathrm{PDH}$ activity have been found in muscle or in blood platelets in cases of spinocerebellar degeneration (Kark and RodriquezBudelli, 1979) and an increase in the state of phosphorylation of $\mathrm{PDH}$ has been described in one instance of encephalomyelopathy (DeVivo et al., 1979). More recently it has been shown that Alzheimer's disease is also accompanied by a decrease in the levels of $\mathrm{PDH}$ activity. Moreover, the severity of the disease was correlated with the amplitude of the decrease in PDH activity (Perry et al., 1980). In view of this evidence, we suggest that a variety of degenerative diseases may be triggered by disturbances in PDH activity (either in total enzyme activity or in the phosphorylation state of its $\alpha$-subunit) followed by changes in the regulation of cytosolic free calcium.

Finally, a similar change in the phosphorylation of $\alpha$ $\mathrm{PDH}$ has been found in another instance of synaptic plasticity, namely, in the long-term potentiation of synaptic transmission which follows brief bursts of highfrequency stimulation delivered to various hippocampal pathways (Browning et al., 1979). There are also some indications that activation of calcium-dependent proteinases might be involved in the generation of permanent alterations in neurotransmitter receptors which have been hypothesized to be responsible for long-lasting increases in synaptic efficacy (Baudry and Lynch, 1980; Baudry et al., 1981). Thus, both long-term potentiation and degeneration may result from the same processes (i.e., alterations in $\mathrm{PDH}$ phosphorylation and activity, elevation in cytosolic free calcium, and the activation of calcium-dependent proteinases). In the first instance, the elevation of calcium levels would be transicnt and the activation of proteinases localized and reversible, resulting in local disturbances in membrane structures and functions. In the case of denervation, the changes in calcium levels would be more lasting, resulting in more generalized changes such as dendritic atrophy and loss of processes, followed later by reactive synaptogenesis (Lynch and Baudry, 1983). Thus, varying degrees of activation of the same enzymatic machinery could produce a continuum of effects from alterations in synaptic efficiency to neuronal degeneration.

\section{References}

Baudry, M., and G. Lynch (1980) Regulation of hippocampal glutamate receptors, evidence for the involvement of a calcium-activated protease. Proc. Natl. Acad. Sci. U. S. A. 77: 2298-2302.

Baudry, M., M. Bundman, E. Smith, and G. Lynch (1981) Micromolar levels of calcium stimulate proteolytic activity and glutamate receptor binding in rat brain synaptic membranes. Science 212: 937-138.

Baudry, M., J. Fuchs, M. Kessler, D. Arst, and G. Lynch (1982a) Entorhinal cortex lesions induce a decreased calcium transport in hippocampal mitochondria. Science 216: 411-413.

Baudry, M., M. Kessler, E. K. Smith, and G. Lynch (1982b) The regulation of pyruvate dehydrogenase activity in rat hippocampal slices: Effect of dichloroacetate. Neurosci. Lett. 31: 41-46.

Becker, G. L., G. Fiskum, and A. L. Lehninger (1980) Regulation of free $\mathrm{Ca}^{++}$by liver mitochondria and endoplasmic reticulum. J. Biol. Chem. 255: 9009-9012.

Browning, M., T. Dunwiddie, W. Bennet, W. Gispen, and G. Lynch (1979) Synaptic phosphoproteins: Specific changes after repetitive stimulation of the hippocampal slice. Science 203: 60-62.

Browning, M., M. Baudry, W. Bennett, and G. Lynch (1981) Phosphorylation-mediated changes in pyruvate dehydrogenase activity influence pyruvate-supported calcium accumulation by brain mitochondria. J. Neurochem. 36: 1932-1940.

Bygrave, F. L. (1977) Mitochondrial calcium transport. Curr Top Bioenerg. 6: 259-318.

Clark, J., and W. Nicklas (1970) The metabolism of rat brain mitochondria. J. Biol. Chem. 245: 4724-4731.

DeVivo, D. C., M. W. Haymond, K. A. Obert, J. S. Nelson, and A. S. Pagliara (1979) Defective activation of the pyruvate dehydrogenase complex in subacute necrotizing encephalomyelopathy (Leigh disease). Ann. Neurol. 6: 483-494.

Erulkar, S. D., and A. Fine (1979) Calcium in the nervous system. Reviews of Neuroscience, Vol. 4, pp. 179-232, Raven Press, New York.

Gall, C., G. Rose, and G. Lynch (1978) Proliferative and migratory activity of glial cells in the deafferented hippocampus. J. Comp. Neurol. 183: 539-550.

Gall, C., R. McWilliams, and G. Lynch (1979) The effects of collateral sprouting on the density of innervation of normal target sites: Implications for theories on the regulation of the size of developing synaptic domains. Brain Res. 175: 37-47.

Gall, C., J. R. McWilliams, and G. S. Lynch (1980) Accelerated rates of synaptogenesis by sprouting afferents in the immature hippocampal formation. J. Comp. Neurol. 193: 10471062.

Happel, R. D., K. P. Smith, N. L. Banik, J. M. Powers, E. L. Hogan, and J. D. Balantine (1981) $\mathrm{Ca}^{2+}$ accumulation in experimental spinal cord trauma. Brain Res. 211: 476-479.

Ishiura, S. (1981) Calcium-dependent proteolysis in living cells. Life Sci. 29: 1079-1087.

Joffe, M., N. Savage, and H. Isaacs (1981) $\mathrm{Ca}^{2+}$-uptake properties of two populations of mitochondria from normal and denervated rat soleus muscle. Biochem. J. 200: 671-677.

Kark, R. A. P., and M. Rodriguez-Rudelli (1979) Pyruvate dehydrogenase deficiency in spinocerebellar degenerations. Neurology 29: 126-131.

Lai, J. C. K., and J. B. Clark (1976) Preparation and properties of mitochondria derived from synaptosomes. Biochem. J. 154: 423-432.

LaManna, J. C., S. I. Harik, A. I. Light, and M. Rosenthal (1981) Norepinephrine depletion alters cerebral oxidative metabolism in the active state. Brain Res. 204: 87-101.

Leiter, A. B., M. Weinberg, F. Isomashi, M. F. Utter, and T. 
Linn (1978) Relationship between phosphorylation and activity of pyruvate dehydrogenase in rat liver mitochondria and the absence of such a relationship for pyruvate carboxylase. J. Biol. Chem. 254: 2716-2723.

Lowry, O. H., N. J. Rosebrough, A. L. Farr, and R. J. Randall (1951) Protein measurement with the Folin phenol reagent. J. Biol. Chem. 193: 265-275.

Lynch, G., and M. Baudry (1983) Origins and manifestations of neuronal plasticity in the hippocampus. In Clinical Neurosciences, W. Willis, ed., Churchill-Livingstone Publishers, in press.

McWilliams, J. R., and G. S. Lynch (1978) Terminal proliferation and synaptogenesis following partial deafferentation. J. Comp. Neurol. 180: 581-615.

McWilliams, J. R., and G. S. Lynch (1979) Terminal proliferation in the partially deafferented dentate gyrus. Time courses for the appearance and removal of degeneration and the replacement of lost terminals. J. Comp. Neurol. 187: 191-198.

Marshall, J. F., J. W. Critchfield, and M. B. Kozlowski (1981) Altered succinate dehydrogenase activity of basal ganglia following damage to mesotelencephalic dopaminergic projections. Brain Res. 212: 367-377.

Mitrius, J. C., D. G. Morgan, and A. Routtenberg (1981) In vivo phosphorylation following ${ }^{32} \mathrm{P}$ orthophosphate injection into neostratum or hippocampus: Selective and rapid labeling of electrophoretically separated brain proteins. Brain Res. 212: $67-81$.

Morgan, D. G., and A. Routtenberg (1981) Brain pyruvate dehydrogenase: Phosphorylation and enzyme activity altered by a training experience. Science $214: 470-471$.

Nemeth, P. M., D. Meyer, and R. A. Pieter Kark (1980) Effects of denervation and simple disuse on rats of oxidation and on activities of four mitochondrial enzymes in type I muscle. J. Neurochem. 35: 1351-1360.

Nicholls, D. G., and I. D. Scott (1980) The regulation of brain mitochondrial calcium-ion transport. Biochem. J. 186: 833839.

Perry, E. K., R. H. Perry, B. E. Tomlinson, G. Blessed, and P. H. Gibson (1980) Coenzyme A acetylating enzymes in Alzheimer's disease: Possible cholinergic comportment of pyruvate dehydrogenase. Neurosci. Lett. 18: 105-110.

Portzehl, H., P. Caldwell, and J. Ruegg (1964) The dependence of contraction and relaxation of muscle fibers from the crab Maia Squinado in the internal concentration of free calcium ions. Biochim. Biophys. Acta 19: 581-591.

Rose, G., C. W. Cotman, and G. S. Lynch (1976) Hypertrophy and redistribution of astrocytes in the deafferented hippocampus. Brain Res. Bull. 1: 87-92.

Schlaepfer, W. W. (1977) Structural alterations of peripheral nerve induced by the calcium ionophore $\mathrm{A}_{23187}$. Brain Res. 176: 1-9.

Schlaepfer, W. W. (1979) Nature of mammalian neurofilaments and their breakdown by calcium. In Progress in Neuropathology, H. Zimmerman, ed., Raven Press, New York.

Schlaepfer, W. W., and S. Micko (1978) Chemical and structural changes of neurofilaments in transected rat sciatic nerve. J. Cell. Biol. 78: 369-378.

Seals, J. R., and M. P. Czech (1980) Evidence that insulin activates in intrinsic plasma membrane protease in generating a secondary chemical mediator. J. Biol. Chem. 255: 65296531.

Seals, J. R., and L. Jarret (1980) Activation of pyruvate dehydrogenase by direct addition of insulin to an isolated plasma membrane/mitochondria mixture: Evidence for generation of insulin's second messenger in a subcellular system. Proc. Natl. Acad. Sci. U. S. A. 77: 77-81.

Stewart, O., and L. K. Smith (1980) Metabolic changes accompanying denervation and reinnervation of the dentate gyrus of the rat as measured by ${ }^{3} \mathrm{H}$-2-deoxyglucose autoradiography. Exp. Neurol. 69: 513-527. 